\title{
Games na terapia de linguagem em crianças com TEA: uma interface com a crise sanitária
}

\section{Games in language therapy in children with ASD: an interface with the health crisis}

DOI: $10.46919 / \operatorname{archv2n3-008}$

Recebimento dos originais: 01/05/2021

Aceitação para publicação: 30/06/2021

\author{
José Danillo dos Santos Albuquerque \\ Graduando em Fonoaudiologia pela Universidade Federal da Paraíba \\ Rua Maria Nely Trajano de Sousa, 148 - João Pessoa-PB \\ E-mail: danilloalbuquerque@hotmail.com \\ Laryssa Moreira Dias. \\ Graduanda em Terapia Ocupacional pela UFPB. \\ Rua Graciliano Delgado, 159- João Pessoa-PB. \\ E-mail: laryssa.moreira.dias@academico.ufpb.br \\ Patrícia Moreira Batista de Souza \\ Fonoaudióloga pela Universidade Federal da Paraíba \\ Centro de Ensino Técnico Odontológico do Nordeste \\ Rua Prefeito Osvaldo Pessoa, 252, Jaguaribe, João Pessoa-PB \\ E-mail: patriciambds@gmail.com

\section{Kaíque de Abreu Cajú} \\ Terapeuta Ocupacional pela Universidade Federal da Paraíba \\ Universidade de Pernambuco - UPE \\ Rua do Riachuelo, 201, Boa Vista, Recife-PE \\ E-mail: kaiquetoabreu@gmail.com
}

\begin{abstract}
RESUMO
O Transtorno do Espectro Autista (TEA) está ligado a problemas na comunicação e interação social, que podem ser identificados e acompanhados por profissionais de saúde capacitados. Devido à pandemia em decorrência do COVID-19, a telessaúde se afrima como forma de dar continuidade a assistência em saúde, e os serious games como ferramenta auxiliar nos processos de intervenção terapêutica. Com o objetivo de investigar o uso de jogos na terapia de linguagem no autismo e como forma de compreender vantagens e desvantagens dessa abordagem terapêutica foi realizada uma revisão integrativa de textos publicados entre o período 2010-2020. Os textos foram buscados por meio de descritores nas línguas portuguesa e inglesa. 9 artigos atenderam a questão de pesquisa. Todos os estudos apontam a linguagem como a melhor maneira de comunicação e mostram os serious games como um meio divertido de intervenção que favorece positivamente o processo terapêutico. Existem trabalhos desenvolvidos na área que resultaram positivamente nas práticas profissionais, melhorando a comunicação e a intervenção entre pacientes e terapeutas.
\end{abstract}

Palavras-chave: Terapia da linguagem, Jogos de vídeo, Transtorno do espectro autista, Saúde pública. 


\begin{abstract}
Autistic Spectrum Disorder (ASD) is linked to problems in communication and social interaction, which can be identified and monitored by trained health professionals. Due to the pandemic due to COVID-19, telehealth is affronted as a way to continue health care, and serious games as an auxiliary tool in the processes of therapeutic intervention. In order to investigate the use of games in language therapy in autism and as a way to understand the advantages and disadvantages of this therapeutic approach, an integrative review of texts published between 2010-2020 was carried out. The texts were searched using descriptors in Portuguese and English. 9 articles answered the research question. All studies point to language as the best way of communication and show serious games as a fun way of intervention that positively favors the therapeutic process. There are works developed in the area that have resulted in positive professional practices, improving communication and intervention between patients and therapists.
\end{abstract}

Keywords: Language therapy, Video games, Autism spectrum disorder, Public health.

\title{
1 INTRODUÇÃO
}

O Transtorno do Espectro Autista (TEA) é uma síndrome neurodesenvolvimental complexa, que leva a sintomas que incluem prejuízos na comunicação e interação social, interesse restrito e comportamento repetitivo que são facilmente detectáveis na primeira infância ${ }^{1}$. Também podem existir comprometimentos nas habilidades motoras das pessoas com TEA, no que diz respeito ao planejamento e sequenciamento motor global e na fala ${ }^{2}$. Dentro da equipe multiprofissional, os profissionais Fonoaudiólogos e Terapeutas Ocupacionais costumam estar na "linha de frente" no processo terapêutico, principalmente no que se refere a estimulação precoce $^{3}$, e neste estudo, terão seus olhares e atuação evidenciados.

A fonoaudiologia é a ciência que estuda, lida diretamente e oferece tratamento para alterações na comunicação. Existem várias áreas de atuação, permitindo conversas interprofissionais diversas, dentre elas: voz, motricidade orofacial, fonoaudiologia hospitalar, fonoaudiologia escolar, saúde coletiva e linguagem ${ }^{4}$.

A linguagem é uma das áreas da fonoaudiologia com mais perspectiva de demanda e mercado de trabalho. Lida com as questões linguísticas em aspectos subjetivos e objetivos ${ }^{5}$. Dentre as demandas em ambulatórios, consultórios e demais cenários de atuação, estão os Transtornos da fala, Transtorno do Desenvolvimento da Linguagem ${ }^{6}$, e os Atrasos no Desenvolvimento da Linguagem, estes, muito comuns em casos de Autismo?

A Terapia Ocupacional é um campo de conhecimento e intervenção em saúde, em educação e na área social. Os profissionais utilizam de forma terapêutica as atividades diárias (ocupações) com o propósito de possibilitar ou melhorar a participação de indivíduos em seus papéis, hábitos e rotinas. A profissão reúne tecnologias orientadas para a emancipação e autonomia de pessoas que, devido a problemáticas específicas (físicas, sensoriais, psicológicas, mentais ou sociais, apresentam dificuldades de inserção e participação na 
vida social temporária ou definitivamente ${ }^{8}$.

No Brasil, a Terapia Ocupacional tem atuado junto à população com TEA, no sentido de favorecer o desenvolvimento, inclusão social e autonomia ${ }^{9}$. O terapeuta ocupacional possui ferramentas importantes para implementar e desenvolver ações nos contextos de desenvolvimento da criança ou adolescente com autismo, como a casa e outros equipamentos e situações em que ele está inserido ${ }^{10}$.

Vale ressaltar que no tratamento da pessoa com TEA não existe uma única abordagem que consiga abarcar toda a complexidade envolvida, e por isto, para alcançar o objetivo de minimizar suas dificuldades, ampliar suas potencialidades e facilitar a vida cotidiana e o cuidado de forma integral, faz-se necessário, além da troca interdisciplinar com outros profissionais e acompanhar as necessidades de acordo com cada faixa etária, acolher e incluir a família, sem exigir dela o papel de terapeuta ${ }^{11}$.

De acordo com a realidade vivida em nosso país, nos serviços de saúde e com o surgimento do COVID-19, a telessaúde e as demais formas de tecnologia remotas se colocaram no cenário brasileiro e internacional, e dessa forma, tornou-se importante mostrar as aplicações existentes na temática aos pacientes, familiares e profissionais de saúde ${ }^{12}$.

Alguns autores já começaram a se preocupar com a qualidade do atendimento e ensino voltado para crianças autistas. Carvalho Filha et al., (2020) fizeram um levantamento de aplicativos direcionados ao processo de ensino e aprendizagem de crianças com TEA, e constataram que o uso de aplicativos em tablets, smartphones e computadores são fortes e potenciais aliados no processo de desenvolvimento de crianças com TEA ${ }^{13}$. O uso de atividades presentes nesses ambientes tecnológicos servem para motivar as crianças a iniciarem tais atividades nos meios tecnológicos e assim, conseguirem expandi-las ao contato real com outras pessoas, criando a partir daí vínculos afetivos e interação social com o meio em que se está inserido.

Os jogos digitais podem ser definidos como sendo uma atividade complexa e completa, mas lúdica. Esses jogos são capazes de conduzir uma terapia e auxiliar os profissionais de saúde; os desfechos desconhecidos motivam os jogadores e todo o desempenho destes, depende exclusivamente de si, desde que o comando tenha sido bem interpretado ${ }^{14}$.

Podemos definir aplicativos (Apps) como sendo ferramentas utilizadas por meio de dispositivos móveis como celulares e tablets, onde seu sistema operacional e recoberto de software ${ }^{15}$. Já os serious games são aplicações que levam o usuário a realizar determinada ação com propósito de atingir algum objetivo $^{16}$.

De forma indireta, os games e a linguagem estabelecem uma comunicação desde a época de Piaget e Vygotsky ${ }^{14}$. Os jogos virtuais beneficiam seus pacientes através de saúde, entretenimento, treinamento e educação, por exemplo ${ }^{17}$. Não podemos esquecer ainda, que o uso de tecnologias com essas crianças ainda é limitado devido às adaptações que precisam ser realizadas - como a tradução - para atingir o público ${ }^{18}$.

O teleatendimento ganhou cada vez mais espaço em todos os cenários de saúde. Mas como a terapia 
interdisciplinar, principalmente no que se refere a estimulação de habilidades relacionadas a transtornos do neurodesenvolvimento estão acontecendo nesse período (e vão acontecer cada vez mais durante e após o período pelo qual o mundo está passando), com foco no desenvolvimento da linguagem funcional?

Sendo assim, o objetivo deste estudo é de realizar uma revisão integrativa da literatura, investigando a respeito do uso de jogos na terapia de linguagem no autismo e quais existem, a fim de comparar os achados de tais estudos e, desta forma, melhor compreender as vantagens e desvantagens desse tipo de abordagem, discutindo as abordagens terapêuticas, adesão do paciente e eficácia no desenvolvimento global de pessoas com TEA. A relevância do estudo se dá a partir do momento que entendemos que a telessaúde é uma realidade que veio para ficar, e mesmo que a crise sanitária seja controlada, a sociedade tenderá a se adaptar a este modelo de assistência à saúde e levará adiante esta prática.

\section{MATERIAL E MÉTODO}

Esta pesquisa foi norteada pela seguinte questão: "Há algum jogo usado nas terapias de linguagem frente ao autismo e quais as características desses?" A temática foi escolhida, pois, realizando o levantamento, constatou-se a carência de estudos mais específicos voltados ao público infantil, especificamente em termos de recursos terapêuticos para o público abordado.

Após definição da questão principal da pesquisa, foram elencadas algumas questões que guiaram a construção das tabelas, o que possibilitou maior visibilidade frente os achados, característicos da metodologia integrativa da literatura e posterior discussão.

Diante disso, a etapa seguinte culminou em determinar as bases de dados que nos serviriam de fonte de informação, para que pudéssemos selecionar os artigos que se adequassem a nossa questão norteadora, selecionando assim, o que fosse de nosso interesse. A busca e pesquisa foi realizada entre os meses de maio e julho de 2020.

Os artigos utilizados foram pesquisados nas seguintes bases: Scientific Electronic Library Online (SciELO), Medical Literature Analysis and Retrievel System Online (MEDLINE), PubMED, Literatura Latino-Americana e do Caribe em Ciências da Saúde (LILACS) e Institute of Eletrical and Eletronic Engineers (IEEE), combinados de forma aleatória, mediante o uso dos descritores em português: linguagem AND jogos, linguagem AND jogos sérios e linguagem OR autismo AND jogos e os descritores na língua inglesa: language AND games, language AND serious games e language OR autism AND games.

Como critérios de inclusão, foram considerados os artigos de texto completo e gratuito, publicados entre 2010 e 2020, que envolvessem crianças, nas línguas portuguesa e inglesa acerca de games que são usados nas terapias de linguagem no autismo. Os artigos que se repetiram nas bases de dados foram excluídos. Após a pesquisa inicial e cruzamento dos descritores nas bases de dados: 0 (zero) artigos na SciELO, 187 (cento e oitenta e sete) na PubMED, 0 (zero) na LILACS, 0 (zero) na MEDLINE e 1 (um) na 
IEEE; realizou-se uma triagem com base nos títulos e os resumos dos artigos e permaneceram 97 (noventa e sete) do PubMED e 1 (um) no IEEE. Em seguida, foi feita uma análise criteriosa, rejeitando aqueles que não preencheram os critérios de inclusão, dessa forma, permaneceram 31 (trinta e um) artigos. Esses foram lidos integralmente, sendo nove artigos selecionados para compor a amostra do presente trabalho, 8 (oito) da PubMED e 1 (um) da IEEE.

Foi feita a exposição dos resultados obtidos através de síntese dos dados e elaboração de resumos. Desenvolvemos quadros, que posteriormente passaram a ser tabelas, com aqueles artigos que fizeram parte da nossa amostra. Em conjunto, os escritores definiram como possíveis dados a serem levantados: título da obra, autores, periódico/ano, características do estudo, resultados e conclusão.

\section{RESULTADOS}

Após levantamento bibliográfico, os autores identificaram os seguintes aspectos: todos os trabalhos encontrados e utilizados eram artigos de texto completo e todos eles faziam relação direta entre o desenvolvimento/ estímulo da linguagem e funcionalidade em crianças diagnosticadas com TEA.

Tabela 1. Distribuição dos artigos encontrados para realização do trabalho.

\begin{tabular}{llll}
\hline $\begin{array}{l}\text { Base } \\
\text { dados }\end{array}$ & $\begin{array}{l}\text { Artigos } \\
\text { encontrados }\end{array}$ & $\begin{array}{l}\text { Artigos excluídos por não se encaixarem nos critérios } \\
\text { de inclusão }\end{array}$ & $\begin{array}{l}\text { Artigos } \\
\text { selecionados }\end{array}$ \\
\hline SciELO & 0 & - & - \\
\hline PubMED & 187 & 179 & - \\
\hline Lilacs & 0 & - & - \\
\hline Medline & 0 & - & 1 \\
\hline IEEE & 1 & 0
\end{tabular}

A linguagem funcional (envolvendo também aspectos de fala) pode ser considerada uma das principais habilidades prejudicadas no autismo ${ }^{1}$. É através da linguagem que o sujeito se constitui e ocupa um lugar na sociedade ${ }^{19}$. Sabendo disso, e a linguagem estando em déficit, as outras habilidades citadas anteriormente estarão também em desvantagem. Logo, todos os artigos aqui listados tratam direta, ou indiretamente de uma intervenção na área da linguagem. Observe o quadro 1:

Quadro 1. Distribuição técnica dos estudos segundo título do artigo, autores e revista/ ano.

\begin{tabular}{|l|l|l|l|}
\hline Número & Título da Obra & Autores & Revista/ Ano \\
\hline
\end{tabular}




\begin{tabular}{|c|c|c|c|}
\hline 1 & $\begin{array}{l}\text { Using computerized games to teach face } \\
\text { recognition skills to children with autism spectrum } \\
\text { disorder: the Let's Face It! Program. }\end{array}$ & $\begin{array}{lr}\text { TANAKA, } & \text { J.W.; } \\
\text { WOLF, J. } & \text { M.; } \\
\text { KLAIMAN, } & \text { C.; } \\
\text { KOENING, k. et al. }\end{array}$ & $\begin{array}{l}\text { Journal of Child } \\
\text { Psychology and } \\
\text { Psychiatry, } 2010 .\end{array}$ \\
\hline 2 & $\begin{array}{l}\text { Using Multitouch Collaboration Technology to } \\
\text { Enhance Social Interaction of Children with High- } \\
\text { Functioning Autism. }\end{array}$ & $\begin{array}{l}\text { GAL, E.; LAMASH, } \\
\text { L.; BAUMINGER- } \\
\text { ZVIELY, } \quad \text { N.; } \\
\text { ZANCANARO, M. } \\
\text { et al. }\end{array}$ & $\begin{array}{l}\text { Physical \& } \\
\text { Occupational in } \\
\text { Therapy } \\
\text { Pediatrics, 2015. }\end{array}$ \\
\hline 3 & $\begin{array}{l}\text { GOLIAH: A gaming platform for home-based } \\
\text { intervention in Autism - principles and design. }\end{array}$ & $\begin{array}{lr}\text { BONO, } & \text { V.; } \\
\text { NARZISI, } & \text { A.; } \\
\text { JOUEN, } & \text { A-L.; } \\
\text { TILMONT, E. et al. }\end{array}$ & $\begin{array}{l}\text { Frontiers in } \\
\text { Psychiatry, 2016. }\end{array}$ \\
\hline 4 & $\begin{array}{l}\text { Emotiplay': a serious game for learning about } \\
\text { emotions in children with autism: results of a } \\
\text { cross-cultural evaluation. }\end{array}$ & $\begin{array}{lr}\text { FRIDENSON- } & \\
\text { HAYO, } & \text { S.; } \\
\text { BERGGREN, } & \text { D.; } \\
\text { LASSALLE, } & \text { A.; } \\
\text { TAL, S. et al. } & \end{array}$ & $\begin{array}{l}\text { Eur Child Adolesc } \\
\text { Psychiatry, } 2017 .\end{array}$ \\
\hline 5 & $\begin{array}{l}\text { Hand-in-Hand: A communication-Enhancement } \\
\text { Collaborative Virtual Reality System for } \\
\text { Promoting Social Interaction um Children With } \\
\text { Autism Spectrum Disorders. }\end{array}$ & $\begin{array}{l}\text { ZHAO, H.; } \\
\text { SWANSON, A. R.; } \\
\text { WEITLAUF, A. S.; } \\
\text { WARREN, Z. E. et } \\
\text { al. }\end{array}$ & $\begin{array}{l}\text { IEEE } \\
\text { TRANSACTIONS } \\
\text { ON HUMAN- } \\
\text { MACHINE } \\
\text { SYSTEMS, } 2017 .\end{array}$ \\
\hline 6 & $\begin{array}{l}\text { Biofeedback-Based, Videogame Balance Training } \\
\text { in Autism. }\end{array}$ & $\begin{array}{l}\text { TRAVERS, B.G.; } \\
\text { MASON, A. H.; } \\
\text { MROTEK, L. A.; } \\
\text { ELLERTSON, A. et } \\
\text { al. }\end{array}$ & $\begin{array}{l}\text { J } \underset{\text { Autism }}{\text { Dev }} \\
\text { Disord, } 2017 .\end{array}$ \\
\hline 7 & $\begin{array}{l}\text { Teaching Literacy Skills to French Minimally } \\
\text { Verbal School-Aged Children with Autism } \\
\text { Spectrum Disorders with the Serious Game } \\
\text { SEMA-TIC: Na Exploraty Study. }\end{array}$ & SERRET, S. et al. & $\begin{array}{l}\text { Frontiers in } \\
\text { Psychology, } 2017 .\end{array}$ \\
\hline 8 & $\begin{array}{l}\text { A Demonstration Project for the Utility of Kinect- } \\
\text { Based Educational Games to Benefit Motor Skills } \\
\text { of Children with ASD. }\end{array}$ & $\begin{array}{lr}\text { VUKICEVIC, } & \text { S.; } \\
\text { DORDEVIC, } & \text { M.; } \\
\text { GLUMBIC, } & \text { N.; } \\
\text { BOGDANOVIC, } & \text { Z. } \\
\text { et al. } & \end{array}$ & $\begin{array}{l}\text { Perceptual and } \\
\text { Motor Skills, } 2019 .\end{array}$ \\
\hline 9 & $\begin{array}{l}\text { Use of Augmented Reality with a Motion- } \\
\text { Controlled Game Utilizing Alphabet Letters and } \\
\text { Numbers to Improve Performance and Reaction } \\
\text { Time Skills for People with Autism Spectrum } \\
\text { Disorder. }\end{array}$ & $\begin{array}{l}\text { ANTÃO, J.Y.F.L. et } \\
\text { al. }\end{array}$ & $\begin{array}{l}\text { Cyberpsychology } \\
\text { Behavior e Social } \\
\text { Networking, } 2020 .\end{array}$ \\
\hline
\end{tabular}

Fonte: Construção própria dos autores.

O quadro 1 demonstra uma heterogeneidade nos periódicos que publicaram a respeito do tema da revisão, em sua maioria, jornais de psicologia e psiquiatria. O quadro 2 infere a importância e a contribuição dos estudos que variam em experimentos, estudos clínicos, avaliações transculturais, dentre outros: 
Quadro 2. Questões secundárias ao roteiro de pesquisa.

\begin{tabular}{|c|c|c|c|}
\hline Número & $\begin{array}{l}\text { Características do } \\
\text { Estudo }\end{array}$ & Resultados & Conclusão \\
\hline 1 & $\begin{array}{l}\text { Estudo clínico } \\
\text { randomizado para } \\
\text { testar a usabilidade do } \\
\text { programa Let's Face } \\
\text { It na melhora do } \\
\text { processamento facial } \\
\text { de crianças com TEA. }\end{array}$ & $\begin{array}{l}\text { Os participantes receberam } 20 \text { horas de } \\
\text { treinamento com o programa e } \\
\text { demonstraram melhorias confiáveis nas } \\
\text { habilidades analíticas e holísticas de } \\
\text { processamento facial. }\end{array}$ & $\begin{array}{l}\text { O programa teve êxito e } \\
\text { se mostrou uma } \\
\text { ferramenta de intervenção } \\
\text { eficaz e alternativa de } \\
\text { tratamento, sendo ponte } \\
\text { importante para interação } \\
\text { face a face no mundo real. }\end{array}$ \\
\hline 2 & 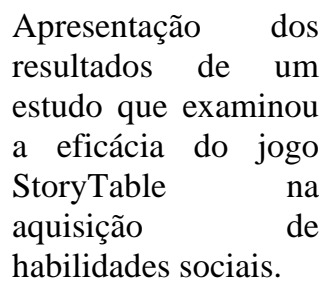 & $\begin{array}{l}\text { Meninos de } 8 \text { a } 12 \text { anos com TEA } \\
\text { passaram por três semanas de intervenção } \\
\text { com sessões de } 45 \text { minutos. Houve } \\
\text { aumento significativo nas taxas de } \\
\text { brincadeiras colaborativas e interações } \\
\text { sociais positivas. }\end{array}$ & $\begin{array}{l}\text { Os resultados sugerem } \\
\text { que o StoryTable fornece } \\
\text { um ambiente rico para } \\
\text { colaboração e melhoria } \\
\text { nas habilidades sociais. }\end{array}$ \\
\hline 3 & $\begin{array}{l}\text { Descrição e teste de } \\
\text { uma plataforma de } \\
\text { jogos desenvolvida } \\
\text { para intervenção } \\
\text { doméstica. }\end{array}$ & $\begin{array}{l}\text { Crianças de } 6 \text { a } 9 \text { anos participaram de } \\
\text { seis sessões semanais, sendo cinco } \\
\text { realizadas em casa e uma no hospital. } \\
\text { Elas apresentaram melhoria no } \\
\text { desempenho em tarefas baseadas em } \\
\text { imitação e atenção conjunta, além de } \\
\text { evolução na autoestima, concentração e } \\
\text { flexibilidade. }\end{array}$ & $\begin{array}{l}\text { O estudo piloto mostrou a } \\
\text { viabilidade } \\
\text { intervenção. }\end{array}$ \\
\hline 4 & $\begin{array}{l}\text { Estudo avaliativo } \\
\text { transcultural do } \\
\text { serious game } \\
\text { Emotiplay nos países } \\
\text { Reino Unido, Israel e } \\
\text { Suécia. }\end{array}$ & $\begin{array}{l}\text { Crianças de } 6 \text { a } 9 \text { anos passaram de oito a } \\
\text { doze semanas de intervenção usando o } \\
\text { serious game. Foi constatado melhor } \\
\text { reconhecimento da emoção e socialização } \\
\text { nas diferentes culturas. }\end{array}$ & $\begin{array}{l}\text { O Emotiplay é um } \\
\text { programa de intervenção } \\
\text { eficaz e motivador para } \\
\text { ensinar o reconhecimento } \\
\text { das expressões. }\end{array}$ \\
\hline 5 & $\begin{array}{l}\text { Desenvolvimento e } \\
\text { avaliação de um } \\
\text { sistema de ambiente } \\
\text { virtual colaborativo. }\end{array}$ & $\begin{array}{l}\text { Participaram } 12 \text { crianças com TEA e seus } \\
\text { pares com desenvolvimento típico. O } \\
\text { sistema funcionou de maneira robusta e } \\
\text { estável, proporcionou comunicação, } \\
\text { colaboração e cooperação entre os } \\
\text { parceiros. }\end{array}$ & $\begin{array}{l}\text { O sistema teve } \\
\text { aceitabilidade das } \\
\text { crianças e demonstrou } \\
\text { potencial de fornecer um } \\
\text { espaço de comunicação } \\
\text { espontânea. }\end{array}$ \\
\hline 6 & $\begin{array}{l}\text { Investigação sobre a } \\
\text { capacidade benéfica } \\
\text { de um treinamento de } \\
\text { equilíbrio visual } \\
\text { biofeedback, através } \\
\text { da combinação de um } \\
\text { novo jogo projetado } \\
\text { com jogos Nintendo } \\
\text { WiiFit prontos. }\end{array}$ & $\begin{array}{l}\text { Crianças e adolescentes com TEA } \\
\text { participaram do treinamento por seis } \\
\text { semanas. Os participantes melhoraram } \\
\text { significativamente os tempos de } \\
\text { equilíbrio, em média, quase dobraram o } \\
\text { tempo que conseguem se equilibrar com } \\
\text { um pé. }\end{array}$ & $\begin{array}{l}\mathrm{O} \text { treinamento de } \\
\text { biofeedback visual foi } \\
\text { eficaz no equilíbrio dos } \\
\text { participantes, sugerindo } \\
\text { que a estabilidade } \\
\text { postural no TEA pode ser } \\
\text { melhorada por meios } \\
\text { além dos tradicionais. }\end{array}$ \\
\hline 7 & $\begin{array}{lr}\text { Investigar } & \text { a } \\
\text { usabilidade do jogo } \\
\text { sério SEMA-TIC } \\
\text { como forma de } \\
\text { aquisição } & \text { de } \\
\text { habilidades } & \text { de }\end{array}$ & $\begin{array}{l}\text { As crianças passaram por um treinamento } \\
\text { de } 23 \text { semanas com o SEMA-TIC. Os } \\
\text { resultados indicam que o serious game } \\
\text { tem usabilidade adequada e que as } \\
\text { crianças adquiriram habilidades de } \\
\text { alfabetização (conhecimento do alfabeto, }\end{array}$ & $\begin{array}{l}\text { Foi confirmada a } \\
\text { possibilidade do acesso ao } \\
\text { aprendizado por meio de } \\
\text { um sistema informatizado } \\
\text { e divertido. }\end{array}$ \\
\hline
\end{tabular}




\begin{tabular}{|c|c|c|c|}
\hline & $\begin{array}{l}\text { alfabetização para } \\
\text { crianças com TEA. }\end{array}$ & $\begin{array}{l}\text { leitura, discriminação, leitura de frases e } \\
\text { segmentação de palavras). }\end{array}$ & \\
\hline 8 & $\begin{array}{l}\text { Projeção e avaliação } \\
\text { de quatro jogos viso } \\
\text { motores baseados em } \\
\text { Kinect, com o } \\
\text { objetivo de avaliar } \\
\text { melhoria } \\
\text { habilidades motoras } \\
\text { de crianças com TEA. }\end{array}$ & $\begin{array}{l}\text { Foram encontradas melhorias } \\
\text { significativas nas habilidades motoras } \\
\text { brutas, além de aumento das emoções e } \\
\text { atenção positivas. }\end{array}$ & $\begin{array}{l}\text { O jogo foi eficaz para } \\
\text { aquisição de habilidades } \\
\text { motoras e provocou } \\
\text { mudanças positivas no } \\
\text { comportamento r e } \\
\begin{array}{l}\text { independência } \\
\text { crianças. }\end{array}\end{array}$ \\
\hline 9 & $\begin{array}{l}\text { Explorar o uso dos } \\
\text { números e das letras } \\
\text { em uma tarefa de } \\
\text { realidade aumentada e } \\
\text { sua influência no } \\
\text { tempo de reação. }\end{array}$ & $\begin{array}{l}48 \text { participantes com TEA e } 48 \\
\text { participantes com desenvolvimento típico } \\
\text { foram recrutados e participaram do } \\
\text { protocolo com o jogo MoviLetrando. Os } \\
\text { resultados apontam melhora no tempo de } \\
\text { reação e estimulação das habilidades } \\
\text { motoras e cognitivas. }\end{array}$ & $\begin{array}{l}\text { Os resultados apoiam a } \\
\text { possibilidade de utilizar o } \\
\text { jogo como forma de } \\
\text { intervenção, podendo } \\
\text { estimular habilidades de } \\
\text { solução de problemas e } \\
\text { empoderamento } \\
\text { cognitivo em crianças } \\
\text { com TEA. }\end{array}$ \\
\hline
\end{tabular}

Fonte: Construção própria dos autores.

\section{DISCUSSÃO}

É vasta a natureza de intervenção e os focos que os jogos sérios (admitidos no estudo) conseguem abarcar e trabalhar em processo terapêutico. Tanaka et al., (2010), pensando em comunicação para além da fala, trabalha com expressões faciais. Pensando no fazer clínico, esse tipo de ferramenta terapêutica pode ser extremamente benéfica pensando na premissa de dar continuidade ao processo terapêutico em casa ${ }^{20}$.

Entretanto, se faz necessário um direcionamento maior para o ambiente familiar, ou seja, a família realizando também esse tipo de intervenção e estimulação com a criança (pois são esses facilitadores na terapia que os profissionais contam durante o período de pandemia). Apesar de saber que uma nova situação de interação será desenvolvida após a pandemia do novo Corona vírus, pensar em habilidades sociais, por exemplo, não pode ficar "de lado" nos planejamentos terapêuticos para crianças com TEA.

Gal et al., (2015) falam sobre habilidades sociais e um ambiente propício ao desenvolvimento das mesmas. O ambiente virtual que o jogo oferece parece ser bastante eficaz no desenvolvimento das habilidades sociais que são imprescindíveis para a colocação social do sujeito. O referido estudo é voltado para autistas com alto grau de funcionamento, sendo bastante eficaz para esse público, assim como existem jogos pensando em habilidades mais básicas para autistas menos funcionais ${ }^{21}$.

A apresentação de uma plataforma de jogos foi feita por Bono et al., (2016) foca nas habilidades de atenção conjunta e imitação se mostrando mais acessível a diferentes públicos ${ }^{22}$, sendo menos focal que o jogo descrito anteriormente ${ }^{21}$. É viável para intervenções domésticas, o que é um fator positivo principalmente no contexto de pandemia. A plataforma parece permitir um processo terapêutico dinâmico, o profissional acompanha o desenvolvimento da criança e realiza as devidas adaptações e ainda permite a 
continuidade da relação terapeuta-paciente, já que podem jogar de maneira compartilhada através da tela de computador.

Outra vantagem da plataforma é o envolvimento da família, os pais jogam e assim participam ativamente da nova forma de terapia. Um fator que deve ser considerado é a individualidade de cada núcleo familiar, devido a possíveis problemas de manuseios da plataforma. Porém, é importante pensar em como adequar esse tipo de ferramenta no contexto escolar, por exemplo, pensando principalmente no novo conceito de normalidade pós-pandemia na realidade brasileira onde as emoções e comportamentos sociais tenderão a mudanças.

Pensando nas emoções, dificuldade frequente, tanto em relação a si, quanto em relação ao outro no autismo, Fridenson-Hayo et al., (2017) trazem um jogo (Emotiplay) voltado às emoções e ao aprendizado das mesmas. A avaliação é feita de forma transcultural e as vantagens disso são as observações dos resultados pelos autores em diferentes realidades e culturas. Isso corrobora o parágrafo anterior, onde falamos da realidade brasileira sob outro aspecto. A cultura interfere diretamente nas emoções e na expressão das mesmas. Portanto, pensar na dinâmica das emoções em diferentes contextos culturais, e mapear as dificuldades enfrentadas por autistas nesses diferentes contextos, pode colaborar com a elaboração de jogos personalizados ${ }^{23}$.

Considerando a importância de conhecer a funcionalidade de cada paciente com TEA, e fundamental a personalização do recurso terapêutico, estudo feito por $^{24}$ colabora com o desenvolvimento da habilidade de cooperação. Essa habilidade se faz imprescindível tanto no ambiente familiar, quanto no escolar e social ${ }^{25}$.

Ainda repercutindo a funcionalidade dos autistas, o estudo realizado por Travers et al., (2017) considera os desafios da estabilidade postural como um impacto negativo no desenvolvimento de uma vida independente. Para beneficiar as pessoas com TEA, produziram um treinamento de equilíbrio visualbiofeedback que através de jogos permite o acompanhamento do equilíbrio e possíveis melhorias. O jogo considera o equilíbrio inicial, promove instruções verbais mínimas e considera as habilidades posturais, sendo um fator positivo por respeitar as características individuais ${ }^{26}$.

Apesar de comprovada a melhoria na estabilidade postural, os jogos apresentam algumas dificuldades de inserção nos planos terapêutico no atual contexto de pandemia. Porque apesar de existir a possibilidade dos jogos serem utilizados em casa e existir o fornecimento de biofeedback contínuo para criança, necessita de um treinamento de uso dos jogos e avaliação postural de maneira presencial. Também, embora tenha sido considerado agradável e benéfico para a família, os jogos parecem não permitir uma interação direta entre a criança e a família.

Ainda buscando o desenvolvimento de habilidades através de jogos, Vukicevic et al., (2019) programaram um conjunto de jogos de realidade virtual focados nas habilidades motoras ${ }^{27}$. Os jogos foram 
eficazes para o objetivo inicial, também se apresentaram benéficos ao aumento das emoções positivas e melhor atenção compartilhada. Um ponto importante é que para jogar a criança precisa ser alfabetizada, para evitar frustração. Dessa forma, o jogo promove crianças mais habilidosas. E todas essas habilidades a serem adquiridas por autistas, interferem na inserção social e contexto familiar (como discutido), por fim, trazemos os jogos sérios que tiveram por objetivo principal, o rendimento escolar.

O SEMA-TIC foi utilizado por crianças francesas, dentro do espectro autista, com grau mínimo de verbalização oral. Foi constatada uma melhora significativa na leitura. O artigo traz a importância dessa habilidade na qualidade de vida em geral do indivíduo com TEA ${ }^{28}$.

A necessidade do mínimo de verbalização para a utilização do jogo sério citado anteriormente, como aliado na alfabetização, infere que, seja lá qual for o recurso (com grau maior ou menor de tecnologia) utilizado com foco escolar, necessita de um feedback auditivo-fonológico, principalmente em crianças autistas com alterações sensoriais e hiporreflexivas ${ }^{29}$.

Assim como Antão et al., (2020), em estudo recente constatou a viabilidade da utilização de realidade aumentada em atividades de letramento ${ }^{30}$. A usabilidade da via visual associada a aprendizagem se mostra proveitosa, para isso, a avaliação de profissionais como o Terapeuta Ocupacional e psicopedagogo, para escolha da melhor via sensorial a ser explorada, faz-se extremamente necessária.

\section{CONCLUSÃO}

Após realização do levantamento bibliográfico, com base na questão de pesquisa, pudemos observar que já existem trabalhos que trazem estudos e resultados sobre esta temática. Os trabalhos encontrados, fazem conexão entre a intervenção convencional e o uso das tecnologias como forma de complemento às práticas profissionais tradicionais.

O público envolvido nos trabalhos, mostram resultados positivos entre as terapias associadas e dessa forma, podemos dizer que há êxito nas intervenções, melhorando a comunicação e interação dos envolvidos; sendo assim, o desenvolvimento de mais serious games nesta área, irão proporcionar maior ganho para os pacientes.

Considerando ainda, que a situação atualmente vivenciada no Brasil e no mundo, aumenta-se a necessidade de se desenvolver este tipo de recurso e disponibiliza-lo para terapeutas e pacientes que tenham a possibilidade e se encaixem em padrões de elegibilidade. Não sendo coerente com segregacionismos, mas aqui é importante deixar claro que nem todas as famílias tem condições de seguir terapias e acompanhamentos de saúde dessa forma. Contudo, a nível de ciência, chega até a ser irresponsável o ato de virar as costas para o rumo da tecnologia que a saúde vem tomando, especialmente após o advento da crise sanitária vivenciada atualmente. 


\section{REFERÊNCIAS}

1. American Psychiatric Association. Diagnostic and statistical manual of mental disorders. Arlington. 2013.

2. SCHMIDT, C. (Org.). Autismo, educação e transdisciplinaridade. Campinas: Papirus, 2013.

3. Moreira NS. O cuidar do portador de autismo e seus familiares: uma abordagem multiprofissional. R. Pesq. Cuid. Fundam. 2010 (Ed. Supl.), 271-274

4. Andrade CRF, Befi-Lopes DM, Lins L. O campo de atuação do Fonoaudiólogo. In: MARCONDES, E.; LINS, L. (Org). Fisioterapia, Fonoaudiologia e Terapia Ocupacional em Pediatria. São Paulo: Savier, 1994

5. Lamônica DAC, Britto DBO. Tratado de linguagem: perspectivas contemporâneas. [S.1: s.n.], 2017.

6. Lima BPS, Guimarães JATL, Rocha MCG. Características epidemiológicas das alterações de linguagem em um centro fonoaudiológico do primeiro setor. Rev Soc Bras Fonoaudiol. 2008;13(4):376-80

7. Zanon RB, Backs B, Bosa CA. Identificação dos Primeiros Sintomas do Autismo pelos Pais. Psic. Teor. e Pesq. 2014. 30(1): 25-33.

8. AMERICAN OCCUPATIONAL THERAPY ASSOCIATION. Estrutura da prática da Terapia Ocupacional: domínio \& processo - $3^{\text {a }}$ ed. traduzida. Revista De Terapia Ocupacional Da Universidade De São Paulo. 2015., v. 26 (esp), p. 1-49.

9. Fernandes A, Santos J. Morato G. A criança com transtorno do espectro autista (TEA). Revista De Terapia Ocupacional Da Universidade De São Paulo. 2018. 29(2):187-94.

10. Minaterl M, Matsukura T. Famílias de crianças e adolescentes com autismo: cotidiano e realidade de cuidados em diferentes etapas do desenvolvimento. Revista De Terapia Ocupacional da Universidade de São Paulo. 2014. 25(2): 126-34.

11. MINISTÉRIO DA SAÚDE. Saúde Mental na Infância e Adolescência. Governo Federal: Florianópolis, 2014. ISBN 85-334-1042-5.

12. Caetano R, Silva AB, Guedes ACCM, Paiva CCN, et al. Desafios e oportunidades para telessaúde em tempos da pandemia pela COVID-19: uma reflexão sobre os espaços e iniciativas no contexto brasileiro. Cad. Saúde Pública. 2020. 36(5): 1-16.

13. Carvalho Filha F, Cardozo BD, Moraes Filho I, Nascimento FL, Silva MV, Pereira M, et al. O uso de aplicativos digitais no processo ensino-aprendizagem de crianças no espectro do autismo: uma revisão integrativa. Revista Enfermagem Atual In Derme. 2020. 91(29): 49-56.

14. Matos ECA, Lima MAS. Jogos eletrônicos e educação: notas sobre a aprendizagem em ambientes interativos. Novas Tecnologias na Educação. 2015. 13(1): 1-10.

15. Biz A, Azzolin R, Nevez AJWA. Estudo dos Aplicativos para Dispositivos Móveis com Foco em Atrativos Turísticos da Cidade de Curitiba (PR). Anais do Seminário da ANPTUR, ISSN: 2359-6805, 2016. 
16. Dias MSC. Jogos sérios para a saúde. Dissertação de Mestrado em Novos Media e Práticas Web Universidade Nova de Lisboa, 2015.

17. Braga M. Realidade virtual e educação. Revista de Biologia e Ciências da Terra. 2001. 1(1): 1-8

18. Fernandes FDM. et al. Computerized resources in language therapy with children of the autistic spectrum. Pró-Fono Revista de Atualização Científica. 2010. 22(4): 415-20.

19. Payer MO. Linguagem e sociedade contemporânea - sujeito, mídia, mercado. 2005. 11(1): 9-25.

20. Tanaka JW, Wolf JM, Klaiman C, Koening K et al. Using computerized games to teach face recognition skills to children with autism spectrum disorder: the Let's Face It! Program. J Child Psychol Psychiatry. 2010. 51(8):944-52.

21. Gal E, Lamash L, Bauminger-zviely, $\mathrm{N}$ et al. Using Multitouch Collaboration Technology to Enhance Social Interaction of Children with High-Functioning Autism. Physical \& Occupational Therapy in Pediatrics. 2015. 36(1): 1-12.

22. Bono V, Narzisi A, Jouen AL, Tilmont E. et al. GOLIAH: A gaming platform for home-based intervention in Autism - principles and design. Frontiers in Psychiatry. 2016. 7(70): 1-16.

23. Fridenson-Hayo S, Berggren D, Lassalle A. et al. Emotiplay': a serious game for learning about emotions in children with autism: results of a cross-cultural evaluation. Eur Child Adolesc Psychiatry. 2017. 26(8): 979-92.

24. Zhao H, Swanson AR, Weitlauf AS. et al. Hand-in-Hand: A communication-Enhancement Collaborative Virtual Reality System for Promoting Social Interaction um Children With Autism Spectrum Disorders. IEEE Transactions on Human-Machine Systems, 2017. 48(2): 136-48.

25. Cabral ABS, Campos ACS, Rodrigues MLB. et al. O uso do PECS como tecnologia do cuidado à criança com autismo. Revista Eletrônica Acervo Saúde. 2019. 31(923): 1-5.

26. Travers BG, Mason, AH, Mrotek, LA. et al. Biofeedback-Based, Videogame Balance Training in Autism. J Autism Dev Disord. 2017. 48(1): 163-75.

27. Vukicevic S, Dordevic M, Glumbic N.; Bognadovic Z. et al. A Demonstration Project for the Utility of Kinect-Based Educational Games to Benefit Motor Skills of Children with ASD. Perceptual and Motor Skills. 2019 0(0):1-28.

28. Serret D. et al. Teaching Literacy Skills to French Minimally Verbal School-Aged Children with Autism Spectrum Disorders with the Serious Game SEMA-TIC: Na Exploraty Study. Frontiers in Psychology. 2017.5(8): 15-23.

29. Miccas C, Vital AAF, Famá ME, et al. Avaliação de funcionalidade em atividades e participação de alunos com transtornos do espectro do autismo. Rev. Psicopedagogia. 2014. 31(94): 3-10.

30. Antão JYFL. et al. Use of Augmented Reality with a Motion-Controlled Game Utilizing Alphabet Letters and Numbers to Improve Performance and Reaction Time Skills for People with Autism Spectrum Disorder. Cyberpsychology Behavior e Social Networking. 2020. 23(1):16-22. 